\title{
«Les Suds profonds de l'Amérique »Photographies de Ralph Eugene Meatyard, Clarence John Laughlin et Alex Harris
}

Pavillon Populaire de Montpellier, du 22 octobre 2010 au 30 janvier 2011 (Commissaire : Gilles Mora)

\section{Jean-Marc Victor}

\section{(Q) OpenEdition}

\section{Journals}

Édition électronique

URL : https://journals.openedition.org/transatlantica/5085

DOI : 10.4000/transatlantica.5085

ISSN : 1765-2766

Éditeur

Association française d'Etudes Américaines (AFEA)

Référence électronique

Jean-Marc Victor, « « Les Suds profonds de l'Amérique »Photographies de Ralph Eugene Meatyard Clarence John Laughlin et Alex Harris », Transatlantica [En ligne], 2 | 2010, mis en ligne le 12 avril 2011, consulté le 07 février 2023. URL : http://journals.openedition.org/transatlantica/5085 ; DOI : https:// doi.org/10.4000/transatlantica.5085

Ce document a été généré automatiquement le 7 février 2023.

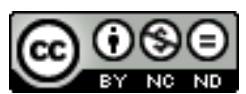

Creative Commons - Attribution - Pas d'Utilisation Commerciale - Pas de Modification 4.0 International - CC BY-NC-ND 4.0

https://creativecommons.org/licenses/by-nc-nd/4.0/ 


\section{«Les Suds profonds de} l'Amérique »Photographies de Ralph Eugene Meatyard, Clarence John Laughlin et Alex Harris

Pavillon Populaire de Montpellier, du 22 octobre 2010 au 30 janvier 2011 (Commissaire : Gilles Mora)

Jean-Marc Victor

1 Cette exposition conçue par Gilles Mora, ${ }^{1}$ récemment nommé directeur artistique du Pavillon Populaire de Montpellier, bel espace entièrement consacré à la photographie, célèbre le $55^{\text {ème }}$ anniversaire du jumelage de la ville avec Louisville, Kentucky. Elle rassemble des œuvres de trois photographes qui, dans des contextes divers et selon des pratiques très différentes, ont porté un regard troublant sur ce « Deep South » dont ils sont issus. Ce faisant, ils en interrogent la nature autant que le rapport à ce(ux) qui l'habite(nt) et peut-être le hante(nt). Tel est, en tout cas, le postulat de cette exposition foisonnante, qui donne lieu à la publication d'un précieux catalogue dont Gilles Mora a rédigé le texte.

2 Sous le titre générique Les Suds profonds de l'Amérique, on découvre en réalité deux expositions en une. D'une part, des images de la Nouvelle Orléans réalisées à un demisiècle d'écart par Clarence John Laughlin (1905-85) et Alex Harris (né en 1949) sont mises en regard, dans l'espace central du rez-de-chaussée du pavillon, sous le titre Nouvelle Orléans: ruines, mythes, chaos. D'autre part, L'Opticien du Kentucky, Ralph Eugene Meatyard propose, dans des salles adjacentes et au premier étage, la première rétrospective française de cet autre sudiste dont les expérimentations formelles ont profondément influencé la scène photographique américaine contemporaine, Cindy Sherman en tête. ${ }^{2}$

3 Dans la première exposition se trouve la série L'œil qui ne dort jamais, ${ }^{3}$ réalisée par Laughlin essentiellement dans les années 1940 et présentée ici en tirages d'époque prêtés par The Historic New Orleans Collection. Après avoir photographié, dans une 
perspective plus factuelle, les vestiges délabrés de l'exubérante architecture de la Nouvelle Orléans, à peine peuplés de rares présences humaines (en particulier des enfants), Laughlin fait poser de mystérieuses silhouettes de femmes voilées prenant des poses altières et hiératiques au milieu des décombres de bâtiments en ruine dans une cité fantôme qui semble en " état de catastrophe permanente » (Mora, 16). ${ }^{4}$ De manière inattendue, l'exposition confronte cette série à celle de Alex Harris intitulée Pèlerinage vers Katrina (visible sur le site du photographe), qui enregistre les traces durables laissées à la Nouvelle Orléans par le passage du cyclone en 2005 dans un style documentaire sobre et distancié, aux antipodes du lyrisme souvent grandiloquent de son prédécesseur. Les photographies de Harris, aux couleurs violentes et volontiers cacophoniques, ${ }^{5}$ qui intensifient l'impact du choc, sont exposées sur fond de palissades en bois qui semblent dire à la fois la précarité et la possibilité de la reconstruction. Audelà de l'unité de lieu (La Nouvelle Orléans), les deux séries font ressentir ce «troptard» inhérent à la saisie photographique de la ruine, voire la prédisposition de la photographie à créer elle-même de la ruine. C'est pourtant bien davantage avec Meatyard, le très lointain voisin du Kentucky, que la filiation de Laughlin est la plus probante, en particulier dans le recours à la mise en scène (voir le site de la Fraenkel Gallery) : l'opticien de Lexington photographie en effet sa famille et ses proches, souvent munis d'accessoires (masques, poupées), sur fond de plantations abandonnées, de jardins en friche, avec lesquels ils entretiennent une relation aussi énigmatique que les femmes spectrales de Laughlin avec leur environnement chaotique.

4 A l'inverse, la présence humaine est rare chez Harris et l'on ne saurait parler, dans son cas, de mise en scène au-delà de l'organisation systématique des clichés en tryptiques. Harris saisit le même lieu de trois points de vue différents par un glissement du regard, le plus souvent de la gauche vers la droite, et capte les effets destructeurs proprement sidérants du cyclone, lesquels frappent bien plus, il faut l'avouer, que « les premières marques du printemps " qu'il dit avoir voulu débusquer plusieurs mois après la catastrophe (Mora, 63). Le principe du tryptique déjoue ici le piège d'une pseudotransparence documentaire puisqu'il inscrit obstinément à l'image la subjectivité changeante du point de vue. Ainsi, par un mouvement ébauchant le déroulement d'une séquence cinématographique, les trois images côte à côte à l'intérieur du même cadre ne captent pas l'effondrement proprement dit (celui-ci a eu lieu, il est déjà trop tard), mais elles en suggèrent l'omniprésence en le démultipliant.

5 Cela dit, il n'y a pas de véritable dialogue formel entre la série de Laughlin et celle de Harris, ce qui n'invalide pas pour autant le choix de cette confrontation entre mise en scène et documentaire, deux versants du médium photographique rarement visibles d'un même coup d'œil dans un champ thématique similaire. Ici, en dehors du «lieu commun » de la Nouvelle Orléans, la visée comparatiste donne à voir ce que le texte introductif de Gilles Mora désigne comme deux avatars de l'effondrement : «Il n'agit plus, dans [le] cas [de Harris], d'un effondrement symbolique, d'un chaos de valeurs menacées. La disparition physique d'une partie de la Nouvelle-Orléans devait solliciter, dans l'urgence, l'enregistrement documentaire, ce qui fut largement réalisé». A l'inverse, cinquante ans plus tôt, Laughlin " construisait " (si l'on ose dire) de toutes pièces le mythe photographique d'une ville comme médusée par sa propre déchéance, engluée dans la nostalgie d'un glorieux passé à jamais perdu, motif sudiste de plus vaste portée, sans cesse ressassé par la littérature et les arts visuels, et théâtralisé ${ }^{~}$ ici avec une emphase qu'on pourra, si l'on veut, encore dire gothique. Un titre résume à lui seul ce désastre programmatique : Mansions into slums (1941). Les insaisissables incarnations 
féminines qui hantent tous ces lieux y seraient, comme le suggère encore Gilles Mora, « figures d'une rédemption possible » ou bien d'un « deuil définitif ».

6 Si l'on veut poursuivre la comparaison à laquelle nous invite le dispositif de l'exposition, ce n'est qu'en jouant sur les mots qu'on peut faire se rejoindre deux approches si dissemblables, c'est-à-dire en plaçant sur le même plan les effets dévastateurs du temps / time (chez Laughlin) et ceux du temps / weather (chez Harris), comme dans un calembour surréaliste assumé. De même, alors que Harris ne donne à ses tryptiques qu'un titre laconique qui précise le lieu de la prise de vue, le plus souvent sous la forme minimale d'une adresse, ${ }^{7}$ la portée symbolique des images de Laughlin, où l'artifice triomphe dans une scénarisation débridée et des poses maniéristes, s'inscrit avec pesanteur dans des titres si longs qu'ils évoquent un surréalisme dont l'humour serait, cette fois, perdu sous les décombres : A Poem of Imprinted Lace. Number Five. A (The) Haunting Gaze. Poem of Lace Shadows. Triumph Over Time - tout cela pour un seul et même cliché. C'est peut-être une faiblesse de telles images que de faire semblant de ne pas pouvoir se passer d'une légende tentaculaire qui les enserre et en restreint le mystère et l'équivoque, comme si la photographie ne pouvait exister pleinement sans le verbe, à l'inverse de ce qui se produit dans les mises en scène de Meatyard, presque toujours dépourvues de titre.

7 C'est à elles qu'en vient l'exposition dans son second temps, de loin le plus enthousiasmant. ${ }^{8}$ On y découvre pour la première fois en France plus d'une centaine de photographies de Meatyard, tirages originaux pour la plupart (à l'exception de quelques tirages plus récents réalisés par l'un des fils du photographe), de petite taille, magnifiquement éclairés, couvrant la quasi-totalité de sa brève carrière, depuis ses premières expériences abstraites à la fin des années 1950, jusqu'à ses derniers autoportraits vibrants à la veille de sa mort en 1972, alors qu'il se savait condamné.

8 La première salle rassemble quelques très beaux exemples des réalisations expérimentales de Meatyard tirées de plusieurs séries où la photographie se donne d'abord comme trace, tournant résolument le dos à quelque forme de mimétisme que ce soit, ce qui semble d'avance donner raison aux interprétations indicielles de l'image photographique qui triompheront dans les années 1980, c'est-à-dire peu de temps après sa mort : absence totale de mise au point donnant naissance à des formes floues et vaporeuses (No-Focus), taches aux contours aléatoires qui évoquent la formation du givre sur une surface plane (Abstractions), littéralisation du sens étymologique de la photographie comme écriture de lumière, où l'appareil saisit les tremblements d'une source lumineuse sur l'eau (Light on Water), réduction extrême de la profondeur de champ ne retenant que le détail exact d'une brindille pour rejeter tout ce qui l'entoure dans un flou où elle flotte mystérieusement et semble, elle aussi, en passe de se dématérialiser (Zen Twigs).

9 Suivent quelques cas de Motion-Sound, troubles et troublants paysages dont le rendu flou provient non d'un bougé du sujet, mais d'un léger déplacement de l'objectif entre plusieurs prises de vue en surimpression, donc légèrement décalées l'une de l'autre, d'où un effet d'étrangeté, comme si le paysage résistait à la saisie photographique en sortant presque de ses gonds. Gilles Mora voit là une manière de « traduire la stridence des paysages du 'Deep South'»; mais ne dirait-on pas que le dispositif crée cette stridence plus qu'il ne la traduit, puisqu'elle n'est pas ici de l'ordre d'une donnée objective prééxistante, mais d'une discordance entre deux occurrences du même, 
irréductibles l'une à l'autre, où le même se déboîte du même et finit par ne plus se ressembler?

10 La troisième salle met à l'honneur les autoportraits avec économie et sobriété. L'un des plus connus parmi les cinq présentés ici juxtapose la silhouette du photographe en chair et en os (« Meat»?) et le mot «Yard» inscrit sur le mur devant lequel il prend la pose, rébus à la fois visuel et textuel qui interroge la substance de la saisie photographique, entre incarnation et fantôme. Un autre le montre montant l'escalier d'une cave, trace humaine sombre et floue, à peine visible en dehors de la main qui empoigne le chambranle de la porte, hésitant entre ancrage et effacement. Dans les trois ultimes autoportraits de 1972, où il apparait en contre-plongée dans l'herbe près d'un arbre mort, il esquisse déjà une sortie toute métaphorique : d'abord couché dans la première image, il est debout dans la seconde et se retourne une dernière fois vers nous, avant de s'enfoncer dans la profondeur de la troisième image qui l'« embaume " déjà tout vif, pour reprendre le célèbre mot de Barthes (Barthes, 30). ${ }^{9}$

11 On trouve à l'étage une magnifique sélection de Romances, ces mises en scène au fort potentiel narratif dont les propres enfants de Meatyard sont les principaux acteurs ${ }^{10}$ et qui ont fait sa notoriété posthume, ainsi qu'une bonne moitié des portraits de couples masqués constituant l'inclassable Family Album of Lucybelle Crater, où sa femme Madelyn, affublée d'un masque d'Halloween, pose en compagnie d'un membre de la famille Meatyard ou d'un ami, lui aussi masqué et différent dans chaque cliché, l'un et l'autre étant immanquablement rebaptisés Lucybelle Crater par la légende. ${ }^{11}$ De multiples formes de défamiliarisation sautent ici aux yeux: c'est paradoxalement la présence incongrue de la famille qui rend les ruines plus inquiétantes qu'elles ne le seraient dans le stéréotype gothique (Romances); c'est elle encore qui déjoue les attentes en subvertissant les codes de l'album de famille (Lucybelle Crater). Comme dans ses paysages tremblés, Meatyard force une nouvelle fois le familier à sortir de ses gonds.

12 Ainsi, au terme d'un parcours photographique faits de ruptures plus que d'échos, on ne saurait malgré tout reprocher à cette exposition l'hétérogénéité des «Suds » qu'elle présente et que le pluriel du titre annonce sans ambiguïté. On s'interroge davantage sur le sens de l'épithète qui décrit ces "Suds" comme "profonds", ce qui tend à reconduire un cliché essentialiste pourtant combattu par certaines images montrées ici, qui fait de la ruine l'éternelle fiancée du Sud et du Sud le lieu de prédilection du refoulé pour une nation entière. Or, si cette lecture peut paraître tentante dans le cas de Laughlin, pour qui les avatars du déclin ont un réel potentiel de fascination, elle semble moins satisfaisante dans le cas de Meatyard, ${ }^{12}$ plus intéressé par une réflexion métapoétique sur le médium photographique que par l'insaisissable essence d'un Sud parmi d'autres, lequel sert surtout de toile de fond à cette réflexion. Mais c'est aussi l'un des nombreux mérites de cette exposition que de nous faire apercevoir bien d'autres choses que les Suds qu'elle nous promet. 


\section{BIBLIOGRAPHIE}

BARTHES, Roland, La Chambre claire, Paris, Cahiers du Cinéma / Gallimard / Seuil, 1980.

KELLER, Judith, Ralph Eugene Meatyard, Paris, Phaidon, Coll. 55, 2002.

LAUGHLIN, Clarence John, Ghosts Along the Mississippi (1948), New York, Crown Publishers / Bonanza Books, 1962.

LAWRENCE, John H., Haunter of Ruins : The Photography of Clarence John Laughlin, Boston, Little Brown / The Historic New Orleans Collection, 1997.

MEATYARD, Ralph Eugene, The Family Album of Lucybelle Crater and Other Figurative Photographs (1974), New York, Distributed Art Publishers, 2002.

MORA, Gilles, Les Suds profonds de l'Amérique, Paris, Democratic Books, 2010.

Ralph Eugene Meatyar, New York, International Center of Photography / Göttigen, Steidl, 2004.

RHEM, James, Ralph Eugene Meatyard, Paris, Nathan / HER, Coll. Photo Poche n87, 2000.

\section{NOTES}

1. Co-fondateur des Cahiers de la photographie, directeur artistique des Rencontres d'Arles de 1999 à 2001, Gilles Mora est notamment l'auteur de plusieurs monographies de photographes américains et de La Photographie américaine, 1958-1981. The Last Photographic Heroes, Paris, Seuil, 2007, Prix Nadar 2007. Il est aussi co-auteur, avec Beverly Brannan, de Les Photographes de la FSA, Farm Security Administration: Archives d'une Amérique en crise, 1935-1943 (Paris : Seuil, 2006).

2. Notons qu'à l'occasion de cette rétrospective, la ville de Montpellier a fait l'acquisition de 15 photographies de Meatyard tirées de la série The Family Album of Lucybelle Crater (1969-72).

3. L'expression est de Clarence John Laughlin lui-même.

4. Voir quelques exemples ici : The Masks Grow to Us, 1947 ; The Repulsive Bed, 1941 ; Our Dead Selves Rise, 1960 ; Our Festering Hands Ruin All, 1949 ; The Bat, 1940 ; Where Shall We Go? 1940.

5. La dominante violette de certains tirages est parfois envahissante.

6. L'une des photographies se nomme A Back Yard as a Stage Set (1941).

7. On s'étonne que ces légendes soient omises dans le catalogue de l'exposition alors que celles des deux autres photographes sont fidèlement reproduites.

8. Oubli ou provocation, Brigitte Ollier ne fait même pas état des deux autres photographes dans son article paru dans Libération le 30 décembre 2010 : « Meatyard, un talent flou ».

9. Bel effet scénographique, d'impressionnants agrandissements de ces trois portraits reviennent en guise d'adieu à la fin de l'exposition.

10. Sa femme n'apparaît que sur une minorité de Romances proposées ici.

11. On regrette que les deux photographies où Meatyard se met en scène lui-même avec sa femme, et qui constituent l'entrée et la sortie de la série, soient ici noyées dans la valse indistincte des Lucybelle. De même, la laconique légende "Same» placée sous l'une des photographies de la série perd tout son sens en dehors de la séquence originale de l'album.

12. Historiquement et culturellement, il est d'ailleurs contestable de placer l'état-lisière qu'est le Kentucky dans ledit « Sud profond». 
INDEX

Thèmes : Trans'Arts 